\title{
Addendum: Effects of exposure to endocrine disruptors, burnout, and social support from peers on premenstrual syndrome in nurses
}

Hye Young Chang ${ }^{1}$, SoMi Park ${ }^{2}$

'Division of Nursing, Wonju Severance Christian Hospital, Wonju, Korea

${ }^{2}$ Department of Nursing, Yonsei University Wonju College of Medicine, Wonju, Korea

This addendum was issued to note the statistical information for the $\mathrm{R}^{2}$ change values in Table 5 of the following article:

Chang HY, Park SM. Effects of exposure to endocrine disruptors, burnout, and social support from peers on premenstrual syndrome in nurses. Korean J Women Health Nurs. 2020;26(2):171179. https://doi.org/10.4069/kjwhn.2020.06.18
Received: September 22, 2020

Revised: September 22, 2020

Accepted: September 22, 2020

Corresponding author:

SoMi Park

Department of Nursing, Yonsei University Wonju College of Medicine, 20 Ilsan-ro, Wonju 26426, Korea

Tel: +82-33-741-0388

E-mail: somi@yonsei.ac.kr

Table 5. Factors influencing premenstrual syndrome $(\mathrm{N}=122)$

\begin{tabular}{|c|c|c|c|c|c|c|c|c|c|c|c|c|}
\hline \multirow{2}{*}{ Variable } & \multicolumn{3}{|c|}{ Model 1} & \multicolumn{3}{|c|}{ Model 2} & \multicolumn{3}{|c|}{ Model 3} & \multicolumn{3}{|c|}{ Model 4} \\
\hline & B & $\beta$ & $\mathrm{t}(p)$ & B & $\beta$ & $t(p)$ & B & $\beta$ & $t(p)$ & B & $\beta$ & $t(p)$ \\
\hline Constant & 19.15 & & $5.31(<.001)$ & 15.42 & & $4.25(<.001)$ & 7.13 & & $0.98(.329)$ & -5.02 & & $-0.67(.499)$ \\
\hline Age & -0.12 & -.09 & $-1.10(.271)$ & -0.15 & -.11 & $-1.34(.182)$ & -.148 & -.11 & $-1.47(.144)$ & -0.09 & -.06 & $-0.93(.350)$ \\
\hline Working department $^{+}$ & 4.06 & .22 & $2.46(.015)$ & 4.96 & .26 & $3.08(.003)$ & 4.57 & .24 & $3.14(.002)$ & 4.16 & .22 & $3.05(.003)$ \\
\hline $\begin{array}{l}\text { Family history of } \\
\text { premenstrual } \\
\text { syndrome }^{+}\end{array}$ & & & & 3.736 & .19 & $2.27(.025)$ & 3.07 & .16 & $2.07(.040)$ & 3.70 & .19 & $2.65(.009)$ \\
\hline $\begin{array}{l}\text { Amount of } \\
\text { menstruation }\end{array}$ & & & & 0.02 & .20 & $2.38(.019)$ & 0.01 & .10 & $1.31(.192)$ & 0.01 & .10 & $1.36(.176)$ \\
\hline Emotional labor & & & & & & & 0.59 & .37 & $4.66(.000)$ & 0.43 & .27 & $3.48(.001)$ \\
\hline Support from peers & & & & & & & -0.11 & -.15 & $-1.94(.054)$ & -0.11 & -.15 & $-2.04(.043)$ \\
\hline $\begin{array}{l}\text { Exposure to } \\
\text { endocrine- } \\
\text { disrupting chemicals }\end{array}$ & & & & & & & & & & 0.25 & .32 & $4.18(<.001)$ \\
\hline$F(d f), p$ & & $3.98(1$ & ), 021 & & 5.20( & 3), 001 & & $9.42(5)$ & $<.001$ & & $11.74(6$ & $6),<.001$ \\
\hline Adjusted $\mathrm{R}^{2}$ & & .0 & & & & 12 & & .2 & & & & 38 \\
\hline $\mathrm{R}^{2}$ change & & & & & $.09(6.0$ & $88, .003)$ & & $8(15.3$ & $2,<.001)$ & & $.09(6.4$ & $40, .002)$ \\
\hline
\end{tabular}

${ }^{+}$The dummy variable references were working department (emergency room and intensive care unit) and family history of premenstrual syndrome (yes). 